\title{
Peno-Scrotal Avulsion Injuries: 20 Years Experience At A Tertiary Care Hospital In Punjab, India.
}

\author{
Jayanthi Mala $\mathrm{A}^{1}$,Vijay AE Obed ${ }^{1}$,Abraham G Thomas ${ }^{1}$. \\ ${ }^{1}$ (Department of Plastic Surgery, Christian Medical College, Ludhiana, India)
}

\begin{abstract}
Penoscrotal avulsion injuries unless associated with the other injuries, is rarely life threatening. However it can put enormous physical, sexual and psychological difficulty to the victims ${ }^{l}$. The study was conducted at Christian Medical College, Ludhiana over a 20 year period from 1991 to 2010. Twenty eight patients with peno-scrotal avulsion injury, were enrolled in the study. Based on our experience in managing these patients we propose a classification for "Penoscrotal Avulsion" injuries. This classification, in future, might also be helpful in developing protocols for management of these injuries.
\end{abstract}

Keywords: Avulsion injuries, Classification, Penoscrotum, Penile Reconstruction, Testicular reimplant.

\section{Introduction}

Avulsion of the penoscrotum it varies from various degree of skin avulsion to virtual emasculation ${ }^{2}$. It can further be complicated by the involvement of regional areas like the perineal skin up to rectum and skin over the abdomen. These injuries were reported previously and were popularly known as "power take - off" injuries, where part of the clothing get caught rotating parts of the tractor machine ${ }^{3}$. In a typical Indian farm settings, wearing loose clothing like kacha, lungi or dhoti, that is worn while working with these high powered equipments makes it easy to be caught in the machine, leading to avulsion injuries.

\section{Material and methods}

The study was conducted at Christian Medical College, Ludhiana, a tertiary care hospital in North of India. It is a retrospective study. All patients who had come to our hospital from 1991 to 2010, with peno-scrotal avulsion injury, were enrolled in the study. The data was collected from the patient file from the hospital records. Exclusion criteria were sharp injuries, gunshot injuries and burns of the genitalia. All avulsion type of injuries were included in the study.

\section{Results}

Over a period of 20 years, we admitted 28 patients with peno-scrotal avulsion injuries. The mean age of presentation was 31 years ranging from 8 years to 67 years. Twenty two patients (78\%) gave history of their clothes getting caught in the moving part of the machine. Six patients $(21 \%)$ had avulsion type injuries due to road traffic accidents.

Of all the injuries, $46.4 \%$ were injuries related to farming accidents. Industrial accidents were $32 \%$ and the road traffic accidents were $21 \%$. The mean hospital stay was 14.7 days (range 8 to 41 days).

Table 1. Types of injuries.

\begin{tabular}{|l|c|}
\hline Type of involvement & Number of patients \\
\hline Penile skin avulsion only & 5 \\
\hline Scrotal skin avulsion only & 2 \\
\hline Peno-scrotal skin avulsion & 17 \\
\hline Regional skin involvement & 12 \\
\hline Penile body avulsion or total penile avulsion & 3 \\
\hline Testicular avulsion & 5 \\
\hline Urethral injuries & 5 \\
\hline Associated skeletal injuries & 6 \\
\hline
\end{tabular}

\subsection{Management:}

On admission, except for the six (21\%) patients with associated skeletal injuries, the patients were found to be haemodynamically stable. The 5 (17\%) patients with penile skin avulsion only, the degloved skin was distally attached in 3 patients. The skin was reposited as composite graft in 3 patients, rest were grafted. The take was satisfactory in only one patient. Rest 2 patients in whom take was poor had undergone debridement, saline dressing and grafting. split thickness skin grafting like the other patients.

In two $(7.1 \%)$ patients with scrotal skin avulsion only, the remaining posterior scrotal skin was undermined and mobilized with reduction of raw area, remaining area anteriorly was grafted. The graft take in these patients were excellent. 
Penoscrotal skin avulsion were seen in 17 (60.7\%) patients. In these patients after obliterating surrounding undermined pockets, rest of the areas were skin grafted with satisfactory results. Two patients had associated urethral injuries required supra pubic cystostomy. The regional skin involvement were found in 12 (42\% )patients along with penoscrotal skin avulsion. They had the following distribution.

- Suprapubic skin involvement in $23 \%$

- Perineal skin involvement in $61 \%$

- Anal verge was involved in $23 \%$

- Groin involvement in $15 \%$

- Thigh involvement in $15 \%$

Two patients had to undergo colostomy due to extensive involvement of skin around the anal verge and perineal area. Total penile and urethral avulsion injuries were in $3(10.7 \%)$ patients and out of them 2 patients had undergone Phalloplasty. One patient was lost in follow up due to psychiatric issues. Phalloplasty or Penile reconstruction was done by Ludhiana technique earlier described by AG Thomas. In which $2^{\text {nd }}$ toe denuded of its soft tissue along with its blood supply from dorsalis pedis artery is anastomosed to the radial artery in the forearm. The $2^{\text {nd }}$ toe along with the neourethra is wrapped around by the radial forearm flap. After two weeks, this composite tissue is later on transferred to the recipient site and anastomosed to the femoral artery and vein of the recipient site. Post operatively these patients were on regular follow up, they either complained of stricture or fistula formation in the neourethra and required corrective procedures for the same.

Testicular avulsion was seen in $5(17 \%)$ patients. Four patients had unilateral testicular avulsion with severe crushing of the affected testis. The remaining less damaged testis were implanted medially in the thigh in three patients and in one patient it was replaced in the remaining scrotal remnants. Only one patient had bilateral testicular avulsion, in this patient temporary ectopic replantation of the testicular vessels were done with the radial vessels of the forearm. After 6 weeks it transferred to the perineum by microsurgically and anastomosed to the femoral vessels.Urethral injuries were seen in $5(17 \%)$ patients, out of which 4 patients required suprapubic catheterization in the hospital and one patient presented to us with suprapubic catherisation done elsewhere.Out of 28 patients presented to us, nineteen patients required urethral catheterization. Three patients did not require any urethral intervention.

Associated skeletal injuries were seen in $6(21 \%)$ patients. Pubic rami fractures was the commonest and was found in 5, the most common skeletal injuries. Maxillofacial injuries were found in 3 patients who sustained injuries due to road traffic accidents.

\section{Discussion}

In Punjab the economy is based on agriculture. With rapid industrialization and advancements in the technology, high powered machines are available to make the work easier and faster to increase productivity. Lack of education and poor compliance for safety is the major cause for most of the accidents. These people are less trained to work with the machinery and less importance is given to safety instructions. No prior education or training courses are provided before working on the high powered machines. There is no current data that can give the exact incidence of peno-scrotal injuries.

In our study wearing loose clothing like 'kacha',lungi or dhoti while operating the high powered machines makes them vulnerable to these type of injuries. Hence it was no surprise that almost half of the victims came from agricultural background. Similar observations were reported by Tripathi et $\mathrm{al}^{4}$.

Our hospital, being a multispecialty hospital, receives patients from all kind of industrial, agricultural and road traffic accidents. In 1957, Brown and Fryer first described the power "take-off injury"2. The typical scenario is, industrial machine pulleys, chains, belts and rotatory parts of the machines as responsible for injuries, when the operators loose clothes get caught in the moving or the rotating part of the machine. The severity of the injury depends on the amount of force exerted by the machine. These type of injuries unless associated with the other injuries are rarely life threatening but it poses physical and immense psychological burden to the patients ${ }^{1}$.

Management of these injuries starts with debridement of the devitalized and contaminated tissues under anesthesia and also to achieve acceptable aesthetic and functional result. There are various classifications of urogenital trauma and management protocols ${ }^{5,6}$. Based on our experience in managing these patients we propose a classification for "Penoscrotal Avulsion Injuries". This classification in future might also be helpful in developing protocols for management of these injuries. 
Table 2: Classification of Penoscrotal Avulsion Injuries.

\begin{tabular}{|c|c|}
\hline Type I & Avulsion injuries involving skin only \\
& \\
\hline & Ip - Avulsion of penile skin only \\
Is - Avulsion of scrotal skin only \\
Ips - Avulsion of penoscrotal skin \\
& Ipsr - Avulsion of penoscrotal and regional skin \\
\hline Type II & Avulsion injury involving penile body and urethra. \\
\hline Type III & Avulsion injury involving the testis . \\
\hline$* U$ - is added if Type I and III if associated with urethral injuries.
\end{tabular}

Type Ip - (Penile skin avulsion only) was seen in 5 of our patients (18\%). The skin viability was checked and the same skin was used for covering the penile wound with its skin attachment still intact to the distal part of the penis. The avulsed segment of skin form the penis includes the loose areolar tissue with its subcutaneous veins, the dartos fascia, and the skin as a unit ${ }^{1}$. Take of a composite graft is questionable and hence reposting was done only if the avulsed segment is still attached. In our study, the take of the composite graft was seen in only one patient. Failure rate was $66 \%$. There was an increase in hospital stay and the cost of treatment as compared patients who had undergone grafting primarily. We strongly recommend split thickness skin grafting than the composite graft. Penis need to be held at length with even pressure distributed to achieve adherence of skin graft. Pedicled flaps can be used for temporary "coverage" of penis while awaiting grafting ${ }^{7}$. Flaps are not recommended because it is thick and recover poor sensation.

Type Is - (Isolated scrotal injury) was seen in 2 patients (7\%). The posterior scrotal skin was usually available and could be stretched for closure. If there were uncovered anterior part of the scrotum if present was grafted. This usually gives a good aesthetic result due hairless skin. Use of tissue expanders have been reported by Still ${ }^{8}$. It has the disadvantage of delayed and multiple procedures that are necessitated by the technique.

Type Ips - (Penoscrotal skin avulsion) was found in 17 patients (60\%). Due to extension of Colles fascia from lower abdominal wall upto the scrotum, skin avulsion of penis may also involve the scrotum. The consensus is that to rapidly close the wound by Finical et $\mathrm{al}^{1}$, which is done by mobilizing the surrounding tissues and grafting the remnant areas. The lymphatic drainage from this area is to the perianal region so lymphoedema is rare in these areas even if grafted. Penoscrotal skin avulsion doesn't usually damage the erectile tissue, the spermatic cord or the testicular function. The patients usually regains normal sexual functions.

Type I psr -( Regional skin involvement) was found in 12 patients (42\%). Six patients had anal verge and perineal skin involvement, out of which two patients needed diversion colostomy to prevent soiling of the graft. Other regional skin involved were suprapubic, groin and rarely thighs.

Type II - Penile avulsion was found in 3 patients. (10\%). Alireza Babaei ${ }^{9}$ et al after evaluation of various techniques in his article had concluded that the radial forearm flap is far superior to the other techniques. In our study Phalloplasty or Penile reconstruction at our centre we have been doing through a technique called "Ludhiana technique" earlier described by AG Thomas which is a modification of the radial forearm flap technique. In which $2^{\text {nd }}$ toe denuded of its soft tissue along with its blood supply from dorsalis pedis artery was anastomosed to the radial artery in the forearm. Neourethra was then reconstructed by tube within tube method in the radial forearm flap. The $2^{\text {nd }}$ toe, along with the neourethra was wrapped around by the radial forearm flap. After two weeks, this composite tissue was later on transferred to the recipient site and anastomosed to the femoral artery and vein of the recipient site. In both the cases there was complete flap survival. The most common complications were urethral stricture and fistula formation.

Type III - Testicular injuries in 5 patients (17\%). Orchiectomy was done in very badly avulsed and crushed testis. In our study 4 patients presented with unilateral testicular avulsion. The less affected and intact testis was implanted in the medial side of thigh, firstly it provides blood supply with the preservation of the cord, in a direct line with the testis. Inner thigh skin is thinner and if the patient desires in a later stage a flap can be raised either laterally or inferiorly and can be applied on the undersurface of the testis ${ }^{3}$. The defect of the flap can be closed primarily or grafted. Moore after his intensive work on the reproductive system and various studies thermal influence on spermatogenesis ${ }^{10,11,12}$, also favors implantation of the testis under the thighs. There are various methods to cover the denuded testicles are there in the literature. Reconstruction of the scrotum by tissue expansion, described by Still et. al. ${ }^{8}$ has the disadvantage of time delay. Single stage repair has been described by Conley et $\mathrm{al}^{13}$ to avoid the negative psychological effect the patient faces for losing his sexual apparatus.

In bilateral testicular avulsions, a novel procedure was reported by S. Ramdas \& A. Thomas ${ }^{14}$ where temporary ectopic replantation of testis was done on the forearm with refabrication and subsequent 
orthotopic microsurgical transfer to the perineum. In our study, one patient who had bilateral testicular avulsion underwent this method of transfer.

\section{Conclusion}

Most of the peno-scrotal avulsion injuries seen by us were preventable. There is an intense need for strict adherence to safety regulations. In the present study almost all of these injuries were seen in people who were wearing loose clothing over the lower half (dhoti or lungi) while operating machines. The proposed classification of peno-scrotal avulsion injuries in this study will help in developing treatment protocols and standardized the treatments of these patients in future.

\section{References}

[1]. Finical SJ ,Arnold PG. Care of the degloved penis and scrotum: a 25 year experience. Plast Reconstr Surg,104 (7): 1999,2074-8.

[2]. Genscosmanoglu R, Bilkay U, Alper M,Gurler T, Cagdas A. Late results of split-grafted penoscrotal avulsion injuries. J Trauma,39(6), 1995, 1201-1203.

[3]. Brown JB, Fryer MP. Peno scrotal skin losses, repaired by implantation and free skin grafting: Report of known normal offspring. Ann surg ,145(5), 1957,656-664.

[4]. Tripathi, F.M. et al. Traumatic avulsion of penile and scrotal skin. British Journal of Plastic Surgery 35(30), $1982,302-303$.

[5]. M Rashid, S Sarwar. Avulsion injuries of the male external genitalia: Classification and reconstruction with the customized radial forearm free flap. Br. J Plast Surg,58( 5),2005,585 -592.

[6]. AM Mohr, AM Pham, RF Lavery, A Sifri et al:Management of trauma to the external genitalia: the usefulness of American Association for the Surgery of Trauma organ injury scales. The Journal of Urology , 170(6), 2003,2311-2315.

[7]. Kubacek V. Complete avulsion of skin of penis and scrotum. Br.J Plast Surg,10, 1958,25.

[8]. Still EFII, Goodman RC. Total reconstruction of a two compartment scrotum by tissue expansion. Plast. Reconstr. Surg $85,1990,805$

[9]. Babaei Alireza; Safarinejad, Mohammed Reza. Penile Reconstruction: Evaluation of the most accepted techniques: Urology Journal;Tehran 7(2),2010, 71-8.

[10]. Moore, C.R and W.J. Quick: The Scrotum as a Temperature Regulator for the Testis. Amer.J. Physiol., 68,1924,70.

[11]. Moore, C.R. : Heat Application and Testicular Degeneration: The Function of the Scrotum. Amer. J. Anat., $34,1924,337$.

[12]. Moore, C.R. : Experimental Studies on the Male Reproductive System. Jour. Urology, 65,1951,497.

[13]. Conley JJ. A one-stage for the repair of the denuded penis and testicles. NY State J Med, 56(19), $1956,3014-3016$.

[14]. S Ramdas, A Thomas, SA Kumar. Temporary ectopic testicular replantation, refabrication and orthotopic transfer. Journal of Plastic, Reconstructive \& Aesthetic Surgery, 60(7), 2007, 700 - 703. 